\title{
TROMBOEMBOLISMO PULMONAR
}

\author{
PULMONARY THROMBOEMBOLISM
}

Leila John Marques

Doutora em Pneumologia e médica assistente do Hospital das Clínicas da Faculdade de Medicina de Ribeirão Preto da Universidade de São Paulo. Departamento de Clínica Médica (Pneumologia).

CorResPondênCIA: Leila John Marques. Departamento de Clínica Médica (Pneumologia) da Faculdade de Medicina de Ribeirão Preto. Campus Universitário - CEP 14048-900 - Ribeirão Preto - SP.

MARQUES LJ. Tromboembolismo pulmonar. Medicina, Ribeirão Preto, 31: 257-265, abr./jun. 1998.

RESUMO: Este capítulo objetiva oferecer conceitos básicos e atualizados, ligados ao tromboembolismo pulmonar, visando, principalmente, o aluno da graduação e médicos generalistas.

UNITERMOS: Embolia pulmonar. Tromboflebite. Anticoagulantes.

\section{CONCEITO}

O termo, tromboembolismo pulmonar (TEP), refere-se ao transporte de coágulos sangüíneos, trombos, desenvolvidos em algum local da circulação venosa sistêmica, com posterior impactação dentro de um ou mais ramos da artéria pulmonar. É importante compreender o TEP não como uma desordem isolada, mas, sim, como uma complicação da trombose venosa profunda (TVP).

Essa obstrução circulatória determina sinais e sintomas relacionados com os sistemas respiratório e cardiovascular, exibindo elevada taxa de mortalidade e morbidade, além de um potencial de recorrência.

\section{INCIDÊNCIA}

Uma estimativa da incidência de TEP varia consideravelmente em relação à população estudada, recursos disponíveis e critérios diagnósticos utilizados. No Brasil, não existem ainda dados fidedignos.

Acredita-se que, nos Estados Unidos, mais de quinhentas mil (500.000) pessoas são acometidas pelo
TEP, por ano. Aproximadamente quarenta mil (40.000) destes indivíduos morrem a cada ano, apesar de terem sido corretamente e tratados. A mortalidade, na primeira hora, gira em torno de $11 \%$ e atinge $30 \%$ daqueles nos quais não foi feito o diagnóstico. Associados a esses dados, estima-se em cinco (5) milhões os episódios de TVP, por ano, naquele mesmo país e que um (1) em cada dez (10) pacientes com TVP desenvolva êmbolos. Os números citados são baseados em investigações clínicas e dados de necrópsias, o que faz com que se reconheça uma incidência de TEP muito mais alta do que se imagina.

\section{ETIOLOGIA E PATOGÊNESE}

Conforme foi citado, o TEP não ocorre sem que haja a formação e propagação de trombos, estando, portanto, diretamente relacionado com a TVP. A literatura reconhece as duas patologias como sendo entidades dinâmicas. Suas conseqüências fisiopatológicas revelam uma verdade, em um dado instante, que poderá não ser mais a mesma em estágios seguintes de suas evoluções. 
Outras substâncias, além de trombos, podem embolizar para os pulmões, em diversas situações, como, por exemplo, óleo, líquido amniótico, gás, fragmentos de projetil de arma de fogo, células gordurosas, talco, dentre outros. Entretanto os trombos venosos são de longe os mais comuns. Mais de $90 \%$ de todas as repercussões clínicas de TEP originam-se nas veias profundas dos membros inferiores (veias poplíteas ou veias profundas mais proximais), detectáveis ou não clinicamente. Outros locais, como veias superficiais, prostáticas, uterinas, renais ou, até mesmo, das câmaras cardíacas direitas, também podem ser fontes de êmbolos.

A tríade de Virchow, caracterizada por estase sangüínea, lesão da camada íntima da parede dos vasos e alterações do sistema de coagulação, identifica os principais fatores predisponentes da trombose venosa. (Tabela I). O papel de cada um destes fatores, na gênese da trombose, já foi demonstrado experimentalmente, porém, na clínica, observa-se que a associação dos dois primeiros, freqüentemente, precede o aparecimento da trombose venosa.

As principais condições que sabidamente aumentam o risco de TEP/TVP são: imobilização no leito, pós-operatórios, varizes, episódio anterior de TVP, neoplasias, traumatismos, idade avançada, puerpério, insuficiência cardíaca, arritmias cardíacas, obesidade, desidratação, tipo sangüíneo $\mathrm{O}$ e tabagismo.

No que diz respeito à patogênese, ainda não se compreendem totalmente os mecanismos exatos que iniciam o processo da trombose venosa. Uma agregação de plaquetas, em torno de uma válvula venosa,

\section{Tabela I - Condições clínicas predisponentes da trombose venosa}

Estase

Lesão da parede do vaso

Hipercoagulabilidade
Imobilidade no leito, Repouso prolongado, Anestesia, Insuficiência cardíaca/Cor pulmonale, Trombose venosa prévia, Obesidade, Gravidez, Veias varicosas, Imobilização de membros por gessos e ataduras.

Politraumatismos, Fraturas ósseas, Inflamação, Cirurgias de grande porte, Queimaduras.

Neoplasia, Coagulação intravascular disseminada, Deficiência da antitrombina III, Terapêutica por estrogênio, Anticorpo anticardiolipina, Síndrome nefrótica, Trombocitose essencial, Trombocitopenia induzida por heparina, Doença inflamatória dos intestinos, Hemoglobinúria paroxística noturna, Deficiências das proteínas C e S. como evento inicial, comumente observado, leva à estimulação dos fatores da coagulação, culminando com fibrinólise e/ou organização. A fibrinólise é rápida e processa a dissolução do trombo. No caso de um processamento incompleto, a resolução é terminada através da organização, que incorpora o trombo residual à parede do vaso com pequena área de tecido fibrótico e posterior reendotelização (Figura 1). Em qualquer fase do processo de resolução, pode ocorrer a embolia pulmonar, como complicação da TVP.

Quando um êmbolo se aloja em um ou mais ramos da artéria pulmonar, observam-se alterações pulmonares e hemodinâmicas. De imediato, as congeram diminuição da seção transversa do leito vascular pulmonar, um aumento da resistência vascular e da pressão arterial pulmonar, bem como um aumento do sença de bactérias, da presença de doença pulmona sença de bactérias, da presença de doença pulmonar hemorragia associada a infarto, atelectasia e pneumonia (nos casos de êmbolo séptico ou infecção com infarto pulmonar). A presença do chamado espaço morto pulmonar, ou seja, uma área que é ventilada sem perfusão, tem, como conseqüências, a constricção do parênquima afetado, perda de surfactante alveolar e hipoxemia. A redução do surfactante pode ocorrer de duas (2) a três (3) horas após o episódio embólico, e se torna severa mais tarde, cerca de doze (12) a quinze (15) horas. Atelectasias importantes, com instabilidade alveolar, podem ser detectadas com vinte e quatro (24) a quarenta e oito (48) horas após a embolia. 


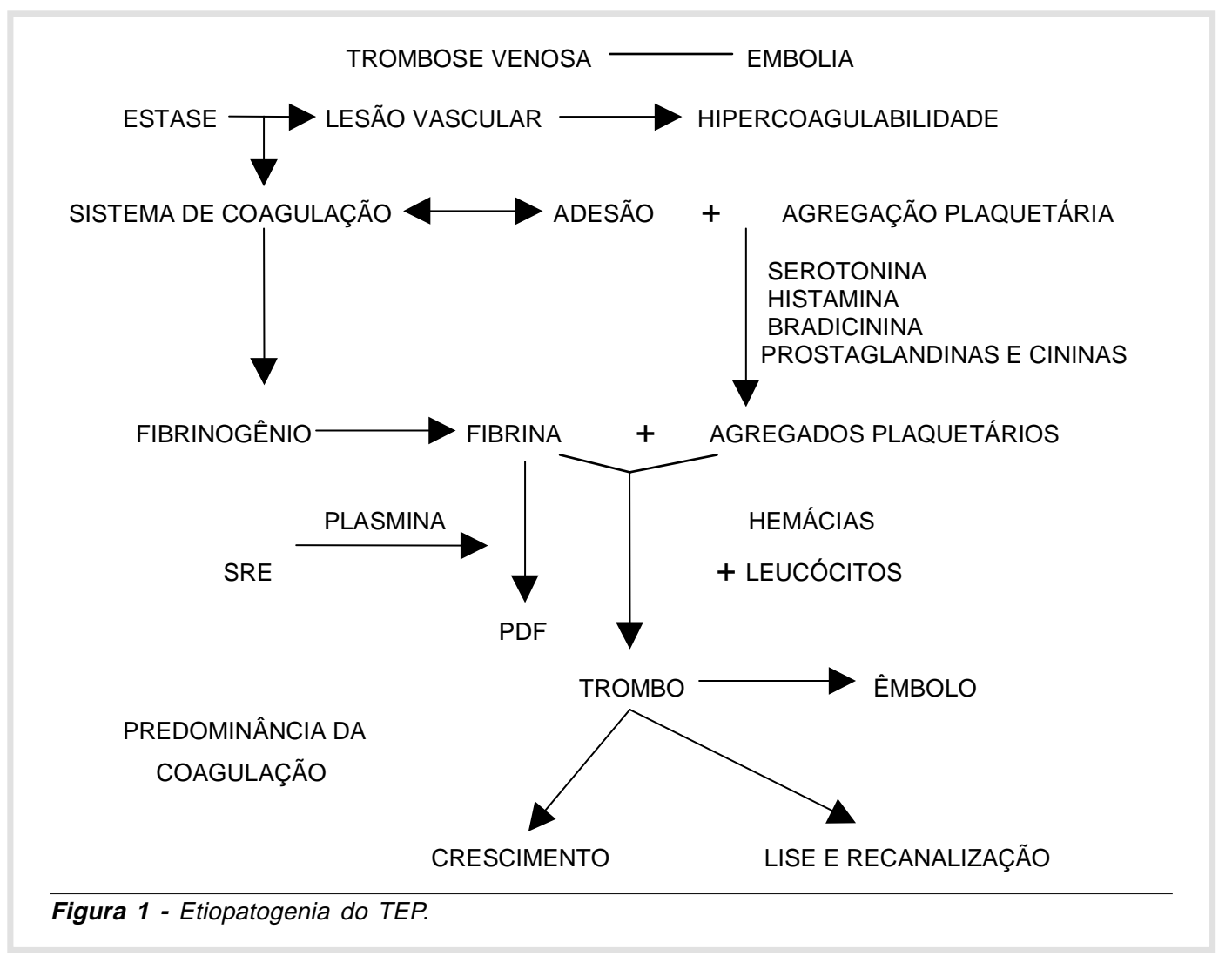

\section{MANIFESTAÇÕES CLÍNICAS}

A grande maioria dos pacientes são assintomáticos ou evidenciam sinais clínicos que só serão reconhecidos retrospectivamente. O diagnóstico clínico do TEP, bem como da TVP, é muito pouco confiável. A clássica dispnéia, de início súbito e inexplicável, sugere o diagnóstico de TEP. Frequientemente, é o único sintoma clínico encontrado. Outras manifestações clínicas, como dor torácica do tipo pleurítica e hemoptise, associadas ao infarto pulmonar, também são sugestivas de TEP, mas não são a regra. A febre, geralmente, nunca superior a 38.5 está presente em 18 a 50\% do casos de TEP com infarto pulmonar. Entretanto, a ausência desses sinais não exclui o diagnóstico de TEP. Além disso, os achados de exame físico são poucos ou inespecíficos. Enquanto a taquicardia ocorre na maioria dos pacientes, outros achados cardíacos, como $\mathrm{B}_{2}$ hiperfonética, $\mathrm{B}_{3}$ de ventrículo direito e íctus de ventrículo direito, só ocorrem em embolias maciças, o que não é o mais freqüente (Tabela II). No exame do aparelho respiratório, sibilos focais e estertores podem ser auscultados, sem serem diagnósticados.

É importante destacar que os achados clínicos, observados no TEP, variam de acordo com: tamanho, número e localização do trombo; oclusão completa ou parcial e presença de doença cardiopulmonar prévia. Naqueles indivíduos com doença cardiopulmonar prévia, a evolução é mais complicada, com a presença mais freqüente de infarto pulmonar, arritmias cardíacas, hipotensão e morte. Já aqueles, considerados "normais antes do episódio", evoluem, freqüentemente, sem sintomas, oligossintomáticos, ou ainda podem evoluir para morte súbita. Cerca de 20 a $30 \%$ dos pacientes com TEP apresentam síncope e parada cardio-respiratória.

Para fins didáticos, costumam-se dividir as formas clássicas de apresentação clínica do TEP em: Maciço e Submaciço, que podem apresentar formas com variados graus de gravidade, ou ainda serem acompanhadas de infarto pulmonar e hipertensão pulmonar. A Tabela II mostra a incidência de sinais e sintomas mais freqüentemente encontrados nas formas de TEP.

A embolia pulmonar maciça ocorre com a obstrução de, no mínimo, 50\% do leito vascular pulmonar. A apresentação clínica é dramática, acompanhada de cor pulmonale agudo, falência cardíaca, hipotensão e choque circulatório. Sinais como dor subesternal severa, arritmias cardíacas, ritmo cardíaco em galope, estase venosa, sopro tricúspide e outros achados, já 
citados, são típicos dessa forma clínica. Entretanto, são raramente documentados, devido à rapidez de instalação do quadro e freqüente evolução dos pacientes para a morte súbita. Felizmente, a forma clínica não é a habitual de se encontrar na rotina. Mais freqüentes são as consideradas grandes embolias, com comprometimento da circulação pulmonar, sem conseqüências fatais. Em muitos casos, a oclusão se faz em menos de duas artérias lobares, com fragmentação periférica dos trombos na árvore vascular. Em tais condições, os pacientes apresentam quadros clássicos de dispnéia, taquipnéia, dor torácica, etc. Dependendo do grau de obstrução e das condições do paciente, os sinais e sintomas variam desde aqueles associados à oclusão maciça até os menos graves, com infarto pulmonar e oclusão periférica.

O TEP com infarto pulmonar pode ocorrer em cerca de $10 \%$ dos episódios de TEP, como conseqüência da tromboembolização periférica para artérias segmentares e subsegmentares. É necessário destacar que infarto não é sinônimo de TEP, mas, sim, que o primeiro é uma complicação rara do segundo. Além da dispnéia súbita, a dor pleurítica aguda e localizada e a hemoptise podem estar presentes, quando ocorre o infarto pulmonar. Sinais clínicos de derrame pleural acompanhado de um quadro febril, simulando uma pneumonia, são encontrados em alguns desses pacientes.

O TEP, seguido de hipertensão pulmonar, ocorre principalmente naqueles casos onde os êmbolos múltiplos de pequeno tamanho obstruem a circulação pulmonar, aumentando a pressão dentro da artéria pulmonar de maneira crescente. Geralmente, está associado a episódios recorrentes de TEP. Sinais clínicos como dispnéia progressiva, falência ventricular direita, arritmia e episódios recorrentes de fibrilação atrial, aparecem, se mais de $50 \%$ do leito vascular estiver comprometido.

Tabela II - Incidência de sinais e sintomas no TEP

\begin{tabular}{lcc}
\hline & TEP maciço (\%) & TEP submaciço (\%) \\
\hline Dispnéia & 85 & 82 \\
Dor torácica pleurítica & 64 & 85 \\
Tosse & 53 & 52 \\
Hemoptise & 23 & 40 \\
Taquipnéia & 95 & 87 \\
Taquicardia (> 100 bpm) & 48 & 38 \\
B2 hiperfonética & 58 & 45 \\
Roncos pulmonares & 57 & 60 \\
Sinais clínicos de TVP & 36 & 26
\end{tabular}

Fonte: Am.J. Med. 62:355-360, 1977.(adaptado)
As manifestações clínicas da TVP são baseadas na inflamação da parede do vaso e na obstrução venosa. A primeira pode levar a dor, hiperestesia, rubor e calor no local da inflamação, enquanto a última acarreta edema, com aumento do diâmetro da região da perna drenada pelas veias envolvidas. A presença do sinal de Homan's, representado pela dor à dorsoflexão do pé, reforça a suspeita de TVP. Entretanto, menos de 50\% dos pacientes com TVP apresentam sinais clínicos que permitam o diagnóstico.

\section{DIAGNÓSTICO}

Os sinais e sintomas clínicos, acompanhados de uma anamnese detalhada, não são suficientes para fazer o diagnóstico de TEP, podendo apenas sugerilo. Alguns testes laboratoriais podem ser úteis na confirmação ou exclusão da suspeita clínica, porém nenhum deles é específico para o diagnóstico de TEP. Leucocitose moderada e elevação da velocidade de hemossedimentação (VHS) estão raramente presentes, a não ser na presença de infarto pulmonar. Um hemograma completo pode ajudar no diagnóstico diferencial, revelando, por exemplo, uma infecção ou uma anemia severa, causas que podem ser responsáveis por uma taquicardia ou uma taquipnéia. Uma série de outros testes, como dosagem de enzimas como a creatinafosfoquinase (CPK) ou dos produtos de degradação da fibrinogênio (FDP), dentre outros, descritos na literatura, não são específicos para o diagnóstico de TEP.

As anormalidades gasométricas, como hipoxemia, hipocapnia, alcalose respiratória, acompanhadas por aumento da diferença alvéoloarterial (gradiente Aa) são comumente associadas à embolia maciça. No entanto, na grande maioria dos casos, o pH arterial, a $\mathrm{PO}_{2}$ e o gradiente A-a são freqüentemente normais ou estão minimamente alterados e, mesmo quando presentes, não são específicos. A presença de uma hipoxemia, sem causa óbvia, aumenta a probabilidade diagnóstica de TEP, sendo necessário prosseguir a avaliação.

O eletrocardiograma (ECG) é normal na maioria dos pacientes ou, geralmente, demonstra uma taquicardia sinusal simples, com alterações não específicas do segmento ST. Apenas na presença de uma sobrecarga ventricular direita, podem-se encontrar os chamados sinais eletrocardiográficos clás- 
sicos do TEP, como padrão $S_{1} Q_{3} T_{3}$, desvio do eixo para a direita, bloqueio do ramo direito e inversão da onda $\mathrm{T}$ nas precordiais direitas. No entanto, o ECG pode não ser diagnosticado e ser útil para afastar outras condições clínicas, como infarto agudo do miocárdio ou pericardite aguda.

A radiografia do tórax (RX) pode ser normal, mesmo na presença de embolia extensa, ou apresentar áreas de hipovascularização (oligoemia ou sinal de Westermark) com ou sem cardiomegalia, redução volumétrica (atelectasia), elevação da hemicúpula diafragmática e derrame pleural. A condensação poderá ocorrer, quando houver transudação hemorrágica com edema ou em zonas de infarto pulmonar. Um padrão de resolução radiológica rápida fala a favor da primeira condição. Nenhuma dessas alterações é específica. A consolidação de uma área do pulmão, associada a um aumento da artéria pulmonar e a uma ou mais densidades em forma de cunha, na periferia do pulmão, com base na pleura (Corcova de Hampton) são achados que indicam TEP com infarto pulmonar (Figura 2). O derrame pleural ocorre em aproximadamente $30-50 \%$ dos pacientes com TEP e, geralmente, está associado ao infarto pulmonar. Vale ainda citar que o RX é importante para excluir outras condições que possam simular as manifestações clínicas mais agudas como, por exemplo, um pneumotórax.

Embora os testes já citados sejam bastante sugestivos e úteis para o diagnóstico diferencial, sabe-se que apenas o mapeamento pulmonar (cintilografia) e a angiografia pulmonar podem, realmente, ser métodos de confirmação diagnóstica.

A maioria das doenças pulmonares não embólicas apresentam um defeito de perfusão, acompanhado por um defeito de ventilação, ao contrário do que ocorre no TEP que, geralmente, leva a distúrbios de perfusão, desacompanhados dos defeitos de ventilação. A cintilografia de ventilação e perfusão é teste não invasivo, fundamentado na presença, extensão e correspondência desses distúrbios (Figura 3). Uma cintilografia de perfusão normal exclui o TEP. Se a visualização da perfusão for anormal, isto é, quando um ou mais defeitos de perfusão estão presentes, deve-se considerar uma variedade de etiologias. Neste caso, uma comparação com a cintilografia de ventilação e com a radiografia de tórax aumentam significativamente as chances diagnósticas.

Uma cintilografia pulmonar de ventilação e perfusão anormal, de alta probabilidade, isto é, com grandes defeitos de perfusão (segmentar ou subsegmentar grande), especialmente quando existem defeitos múltiplos, associados à cintilografia de ventilação normal, indica TEP, na maioria dos casos. Cerca de $40 \%$ a $70 \%$ dos pacientes com suspeita de TEP apresentam o padrão cintilográfico intermediário, chamado "não alta probabilidade", que inclui defeitos de perfusão segmentares ou subsegmentares, grandes, associados a defeitos de ventilação; defeitos de perfusão subsegmentares, pequenos, com ou sem defeitos de ventilação, associados a defeitos de perfusão e a anormalidades no RX.

Embora o mapeamento pulmonar possa ser útil, o único procedimento diagnóstico definitivo, capaz de demonstrar o êmbolo é a angiografia pulmonar. Tal procedimento é invasivo, não é isento de riscos e permite a visualização dos defeitos de enchimento intravascular ou "cut off" do vaso e seu valor depende de sua qualidade e da experiência de quem a interpreta

(Figura 4). Todos os casos suspeitos com cintilografia não diagnóstica merecem uma angiografia. A angiografia também deve ser realizada quando há contra-indicação ao uso de qualquer anticoagulante ou trombolítico, mesmo na vigência de um exame cintilográfico com baixa probabilidade. Esta necessidade pode ainda ser reforçada pela demonstração de uma fonte potencial de êmbolos nas extremidades inferiores, através da pletismografia de impedância, da ultra-sonografia de tempo real ou da venografia. A tomografia computadorizada helicoidal é um método moderno não invasivo, que permite a visualização de trombos intraluminais, com boa especificidade nos casos de TEP importante. Sua utilização, em nosso meio, ainda depende da disponibilidade desse recurso, mesmo nos grandes centros.
Figura 2 - Corcova de Hampton. Hemitórax direito em PA, mostrando velamento homogêneo em topografia de seio costofrênico direito, com contorno convexo em direção ao hilo pulmonar. 


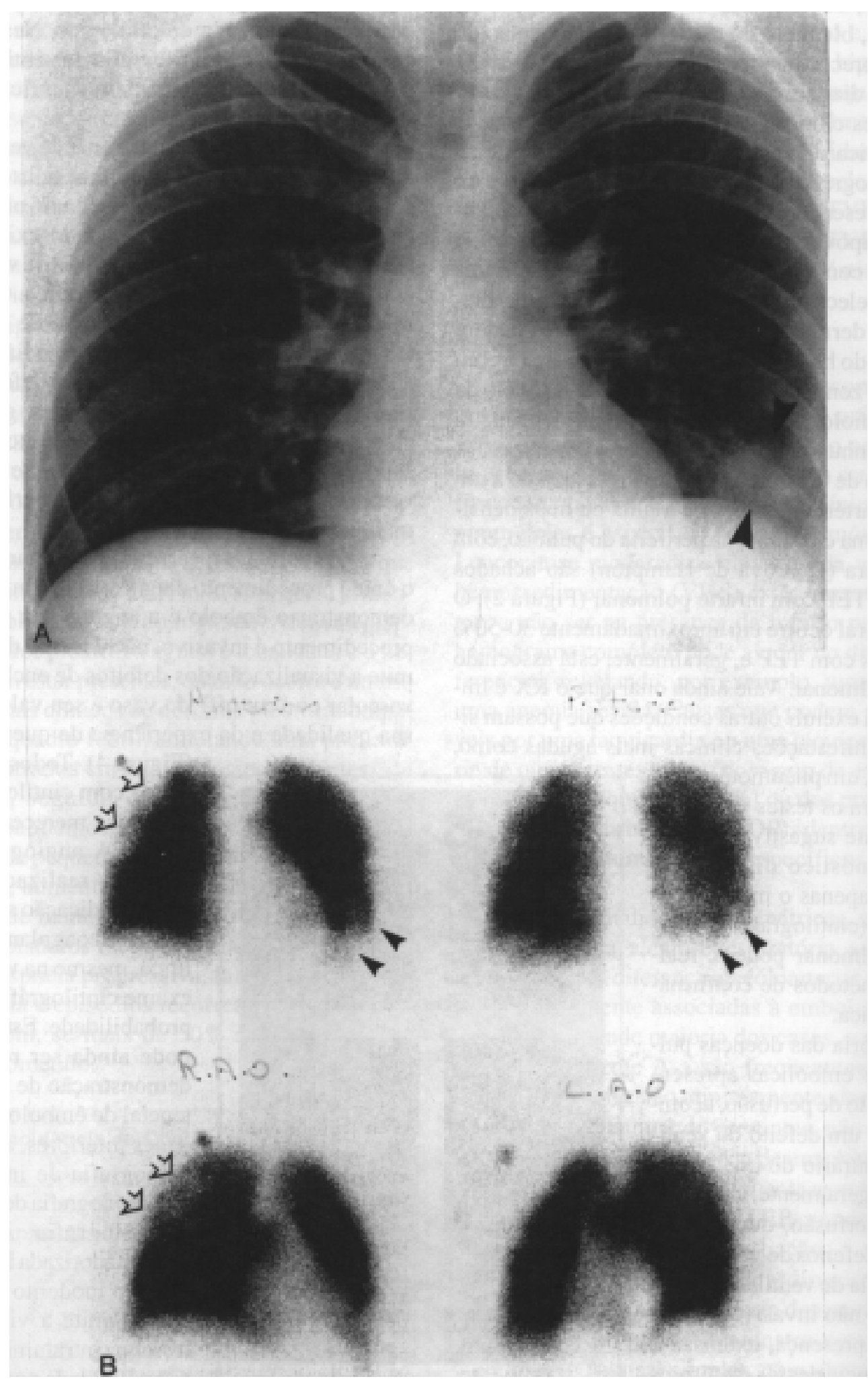

Figura 3 - A) Radiografia de Tórax em PA: opacidade homogênea e arredondada em topografia de lobo inferior esquerdo, elevação da hemicúpula diafragmática esquerda, pulmão direito normal. B) Cintigrafias de perfusão, em projeções anterior, posterior, oblíqua anterior direita e oblíqua anterior esquerda, revelam defeito de perfusão em ambos os pulmões. Destaque maior para lobo superior direito e lobo inferior esquerdo, como mostram as setas. 


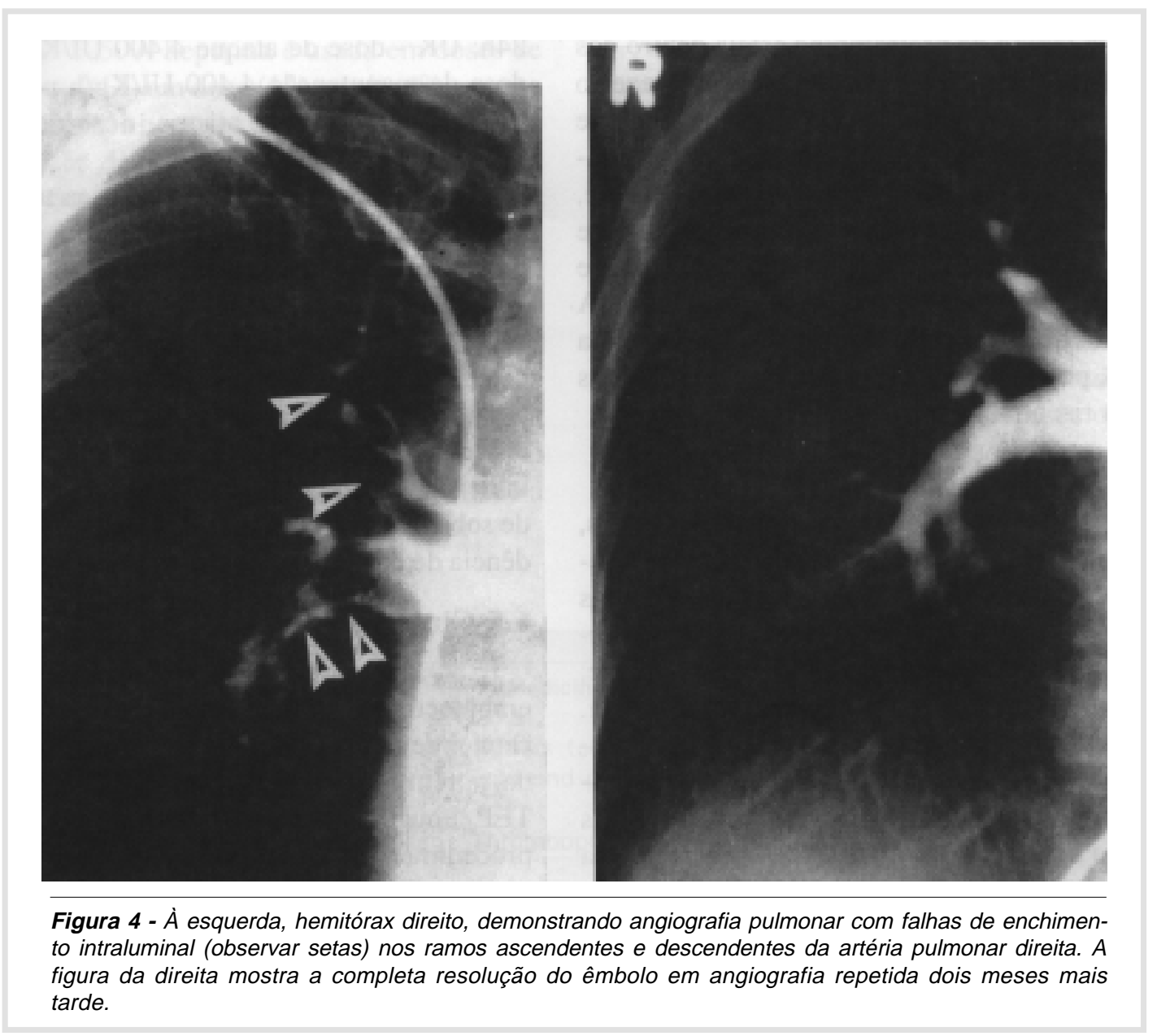

\section{TRATAMENTO}

\subsection{Heparina}

$\mathrm{Na}$ fase inicial, o uso da heparina é essencial. A heparina inibe, de imediato, o crescimento do trombo, apressando a resolução do trombo, reduzindo o tamanho do êmbolo. No entanto, um paciente heparinizado permanece com risco de embolia até que o trombo tenha se dissolvido ou se organizado. Além disso, a heparina inibe a agregação plaquetária que contribui para a liberação de substâncias ativas (serotonina e tromboxane A2), capazes de induzir a vasoconstricção e a broncoconstricção pulmonares. A heparina deverá ser administrada por via endovenosa, na dose inicial de 10.000 a $15.000 \mathrm{U}$, com posterior dose, necessária para manter o tempo de coagulação em duas a três vezes o controle, o que pode ser obtido por infusão contínua de 1.000 a 1.500 U por hora, ou em doses de 5.000 a10.000 U de quatro (4) em quatro (4) horas. Quando o esquema for intermitente, deve-se determi- nar o tempo de coagulação, sempre antes de cada aplicação. O tempo de tromboplastina parcial ativada (TTPA) também pode ser usado no controle da dose de heparina e deve ser mantido 1 e $1 / 2$ a 2 e $1 / 2$ vezes o controle, durante o esquema de infusão endovenosa e 1 e $1 / 2$ vez o controle, imediatamente antes da nova dose, no esquema de administração intermitente. A hemorragia é a complicação principal da heparinização, que deve ser mantida por dez (10) dias.

\subsection{Varfarina}

Um anticoagulante oral deve ser iniciado no quinto ou sétimo dia de heparina, desde que o paciente não tenha desenvolvido nenhum sinal de sangramento excessivo ou queda do hematócrito maior que dez (10) pontos, em cinco dias; caso contrário, manter a heparina por mais tempo ou suspendê-la para novas avaliações. Quando se inicia um anticoagulante oral do tipo varfarina, deve-se atentar para o fato de que um estado antitrombótico efetivo não é atingi- 
do até que o tempo de protrombina esteja dentro dos limites determinados (aproximadamente 1.5 vezes o controle) por, pelo menos, tres (3) ou provavelmente cinco (5) dias. Portanto, a heparina não deve ser retirada antes desse período. A terapia anticoagulante, prolongada deve ser mantida até que os fatores de risco tenham desaparecido(por exemplo: fratura de membro, resolvida com o paciente deambulando). A duração é definida empiricamente e varia de três a seis meses, podendo ser mais extensa para pacientes de elevado risco para trombose recorrente.

\subsection{Trombolíticos}

As justificativas para o uso dos trombolíticos, no TEP são a capacidade de acelerar a lise do coágulo, associada a um rápido bloqueio da formação dos mediadores humorais, melhorando as condições hemodinâmicas e a redução da taxa de TEP recorrente, por dissolver a fonte formadora de êmbolos in situ. A redução da frequiência da hipertensão pulmonar crônica, do risco da síndrome pós-trombose venosa profunda, e a melhor qualidade de vida são vantagens oferecidas a longo prazo. A lise do coágulo se baseia na ativação do plasminigênio, convertido em plasmina, importante enzima do sistema fribrinolítico. Os trombolíticos mais citados na literatura são a estreptoquinase (SK) e a uroquinase (UK), ditos de primeira geração, e o ativador do plasminogênio tecidual (rt-PA), considerado de segunda geração. A SK age, formando um complexo com o plasminogênio ativado, ligado ao coágulo. A UK é um ativador direto do plasminogênio. O rt-PA tem alta afinidade pelo plasminogênio ligado à fibrina, na superfície do coágulo. Ainda não está comprovada uma superioridade de um agente trombolítico sobre os demais.

Embora exista uma tendência atual para ampliar a utilização dos trombolíticos, a rotina, no tratamento do TEP, ainda é restrita aos casos mais graves, como na embolia maciça com cor pulmonale agudo, instabilidade hemodinâmica e grave insuficiência respiratória, baseados na ação rápida destes agentes na reperfusão pulmonar. Nos casos de embolia pulmonar com menores repercussões, acompanhadas de TVP extensa, proximal, seu papel ainda é discutido. Cabe ainda lembrar que essas drogas são caras, implicam em maior risco de hemorragia do que a heparina e devem ser utilizadas por médicos com experiência no seu manejo. Os esquemas terapêuticos mais aceitos atualmente são: SK - dose de ataque: $250.000 \mathrm{UI}$ em $30 \mathrm{~min}$, dose de manutenção $100.000 \mathrm{UI} / \mathrm{h}$ por 24h; UK - dose de ataque $4.400 \mathrm{UI} / \mathrm{Kg}$ em $10 \mathrm{~min}$, dose de manutenção $4.400 \mathrm{UI} / \mathrm{Kg} / \mathrm{h}$ por $12-24 \mathrm{~h}$; rtPA - não há dose de ataque, dose de manutenção $100 \mathrm{mg}$ por $2 \mathrm{~h}$ contínua.

Após o período de terapêutica trombolítica, a anticoagulação padrão se faz necessária para a profilaxia de êmbolos recorrentes.

\subsection{Filtros de veia cava}

A colocação de dispositivos na cava inferior, como o filtro de Greenfield, está indicado nos pacientes com TVP, nos quais a anticoagulação está contraindicada ou, ainda, naqueles com baixa probabilidade de sobrevida a fenômenos de TEP recorrente. A incidência de coágulos, nos filtros, é baixa.

\subsection{Cirurgia}

O papel da cirurgia é bastante discutível. A embolectomia pulmonar, por meio de toracotomia, cateter de fogart ou de sucção, quase nunca é utilizada, porém constitui uma alternativa no tratatmento do TEP. Poucos pacientes são selecionados para esse procedimento, que apresenta alta taxa de mortalidade, quando comparado com as outras condutas não invasivas.

\subsection{Cuidados gerais}

As medidas de suporte cardiopulmonar incluem a administração de oxigênio, caso haja hipoxemia, e tratamento com dopamina, na vigência de hipotensão sistêmica, por falência ventricular direita.

\section{PROFILAXIA}

A prevenção do TEP, que, na verdade, é a prevenção da TVP, inclui, basicamente, cuidados fisioterápicos e instituição medicamentosa que contribuam com a diminuição da formação dos trombos. A movimentação ativa, ou passiva, a elevação dos membros inferiores, massagens, deambulação precoce, uso de meias elásticas e aplicação de ultra-som são medidas importantes na prevenção da TVP. O uso da heparina, em pequenas doses, está indicado nas condições ditas tromboembolígenas de risco de TVP. Dessa forma, a heparina inibe os eventos que ocorrem no início da cascata da coagulação, antes da formação da trombina. $\mathrm{O}$ esquema deve ser iniciado imediatamente após o risco ter sido evidenciado, e continuado até que o mesmo tenha desaparecido. Os melhores resultados são obtidos com atividade trombínica entre $20 \%$ e $30 \%$ 
e INR de 2,5 - 3,5. A heparina é usada em doses de $5.000 \mathrm{U}$ de doze (12) em doze (12) ou oito (8) em oito (8) horas, enquanto a heparina de baixo peso molecular é usada na dose de $7.500 \mathrm{U}$ de $24 / 24$ horas. Nesta dose, a heparina não altera o tempo de coagulação e de sangramento.

Outra opção profilática é a varfarina, sódica, que tem demonstrado ser uma droga eficaz em grupos de alto risco, incluindo pacientes traumatizados, queimados e com cirurgia de quadril, onde as baixas doses de heparina não são eficazes na prevenção da TVP. A droga demora vários dias para agir como antitrombótico, agindo, provavelmente, prevenindo a extensão do trombo ao invés de sua formação.
Drogas como a aspirina e o dipiridamol não demonstram eficácia na profilaxia da TVP.

Finalmente, uma alternativa segura, eficaz e bem tolerada é o uso da compressão mecânica da panturrilha. Tal compressão mantém o fluxo venoso através da insuflação de um "cuff" aplicado sobre a panturrilha, por vários segundos, a cada minuto. Em presença de TVP, ativa está contra-indicado seu uso. Deve ser aplicada imediatamente após a constatação da situação de risco, mantida durante todo o período de risco até que o risco tenha desaparecido. Sua indicação formal é para aqueles pacientes a quem o uso da heparina está contra-indicado como, por exemplo, os pacientes neurocirúrgicos.

MARQUES LJ. Pulmonary thromboembolism. Medicina, Ribeirão Preto, 31: 257-265, apr./june 1998.

ABSTRACT: The aim of this section is to present currents concepts of Pulmonary Thromboembolism for undergrade students and general doctors.

UNITERMS: Pulmonary Embolism. Thrombophebitis. Anticoagulants.

\section{BIBLIOGRAFIA CONSULTADA}

1 - PALEVSKY HI; KELLEY MA \& FISHMAN AP. Pulmonary thromboembolism disease. In: FISHMAN AP, ed. Fishman's pulmonary diseases and disorders. 3th ed. McgrawHill Book, New York, p. 1297-1329, 1998.

2 - FRASER RG et al. Pulmonary thromboembolism. In: FRASER RS ed. et al. Diagnosis of diseases of the chest. 3th ed. W.B. Saunders, Philadelphia, p. 1703 - 1782. 1990.
3 - MOSER KM. Venous thromboembolism. State of the art. Am Rev Respir Dis 141: 235-249, 1990.

4 - BELL WR; SIMON TL \& DEMETS DL. The clinical features of submassive and massive pulmonary emboli. Am J Med 62: 355-361, 1977.

Recebido para publicação em 13/05/98

Aprovado para publicação em 10/06/98 